Article

\title{
Differential Sandwich-Type Results for Symmetric Functions Connected with a $Q$-Analog Integral Operator
}

\author{
Sheza M. El-Deeb ${ }^{1, *, t, \ddagger ~(D)}$, Teodor Bulboacă ${ }^{2}{ }^{\circledR}$ and $\ddagger$ \\ 1 Department of Mathematics, Faculty of Science, Damietta University, New Damietta 34517, Egypt \\ 2 Faculty of Mathematics and Computer Science, Babeş-Bolyai University, 400084 Cluj-Napoca, Romania; \\ bulboaca@math.ubbcluj.ro \\ * Correspondence: shezaeldeeb@yahoo.com; Tel.: +966-0557689615 \\ + Current address: Department of Mathematics, College of Science and Arts in Al-Badaya, \\ Al-Qassim University, Al-Badaya 3803070, Saudi Arabia. \\ $\ddagger$ These authors contributed equally to this work.
}

Received: 5 November 2019; Accepted: 28 November 2019; Published: 2 December 2019

check for updates

\begin{abstract}
In this paper, we obtain some applications of the theory of differential subordination, differential superordination, and sandwich-type results for some subclasses of symmetric functions connected with a q-analog integral operator.
\end{abstract}

Keywords: symmetric functions; Hadamard (convolution) product; differential subordination; differential superordination; sandwich-type results; integral operator

MSC: 30C45; 30C80

\section{Introduction}

The theory of $q$-analysis has an important role in many areas of mathematics and physics. Jackson [1,2] was the first that gave some application of $q$-calculus and introduced the $q$-analog of derivative and integral operator (see also [3]). Let $\mathcal{H}(\mathbb{U})$ denote the class of analytic functions in the open unit disk $\mathbb{U}:=\{z \in \mathbb{C}:|z|<1\}$, and $\mathcal{H}[a, m]$ denote the subclass of functions $f \in \mathcal{H}(\mathbb{U})$ of the form

$$
f(z)=a+a_{m} z^{m}+a_{m+1} z^{m+1}+\ldots, z \in \mathbb{U},
$$

with $a \in \mathbb{C}$ and $m \in \mathbb{N}:=\{1,2, \ldots\}$.

In addition, let $\mathcal{A}(m)$ denote the subclass of functions $f \in \mathcal{H}(\mathbb{U})$ of the form

$$
f(z)=z+\sum_{k=m+1}^{\infty} a_{k} z^{k}, z \in \mathbb{U}
$$

with $m \in \mathbb{N}$, and let $\mathcal{A}:=\mathcal{A}(1)$.

We define the integral operator $\mathcal{K}_{n, m}^{\alpha}: \mathcal{A}(m) \rightarrow \mathcal{A}(m)$, with $\alpha>0$ and $n \geq 0$, as follows:

$$
\mathcal{K}_{n, m}^{0} f:=f,
$$

and

$$
\mathcal{K}_{n, m}^{\alpha} f(z):=\frac{(n+1)^{\alpha}}{\Gamma(\alpha) z^{n}} \int_{0}^{z} t^{n-1}\left(\log \frac{z}{t}\right)^{\alpha-1} f(t) d t
$$


where all the powers are the principal ones, and $\log 1=0$.

If $f \in \mathcal{A}(m)$ has the power expansion of the form in Equation (1), it can be easily verified that

$$
\mathcal{K}_{n, m}^{\alpha} f(z)=z+\sum_{k=m+1}^{\infty}\left(\frac{n+1}{n+k}\right)^{\alpha} a_{k} z^{k}, z \in \mathbb{U} .
$$

For $0<q<1$, the $q$-derivative of the operator $\mathcal{K}_{n, m}^{\alpha}$ is defined by

$$
\partial_{q} \mathcal{K}_{n, m}^{\alpha} f(z):=\frac{\mathcal{K}_{n, m}^{\alpha} f(q z)-\mathcal{K}_{n, m}^{\alpha} f(z)}{z(q-1)}, z \in \mathbb{U},
$$

that is

$$
\partial_{q}\left[z+\sum_{k=m+1}^{\infty}\left(\frac{n+1}{n+k}\right)^{\alpha} a_{k} z^{k}\right]=1+\sum_{k=m+1}^{\infty}\left(\frac{n+1}{n+k}\right)^{\alpha}[k, q] a_{k} z^{k-1}, z \in \mathbb{U}
$$

where

$$
[k, q]=\frac{1-q^{k}}{1-q}=1+\sum_{i=1}^{k-1} q^{i}, \quad[0, q]=0,
$$

It is easily to verify from Equation (2) that

$$
z \partial_{q} \mathcal{K}_{n, m}^{\alpha} f(z)=z+\sum_{k=m+1}^{\infty}\left(\frac{n+1}{n+k}\right)^{\alpha}[k, q] a_{k} z^{k}, z \in \mathbb{U} .
$$

For any non negative integer $k$, the $q$-number shift factorial is given by

$$
[k, q] != \begin{cases}1, & \text { if } \quad k=0 \\ {[1, q][2, q][3, q] \ldots[k, q],} & \text { if } \quad k \in \mathbb{N}\end{cases}
$$

while the $q$-generalized Pochhammer symbol for $r>0$ is defined by

$$
[r, q]_{k}=\left\{\begin{array}{lll}
1, & \text { if } & k=0 \\
{[r, q][r+1, q] \ldots[r+k-1, q],} & \text { if } & k \in \mathbb{N}
\end{array}\right.
$$

For $\lambda>-1$, we define the operator $\mathcal{N}_{n, m, q}^{\lambda, \alpha}: \mathcal{A}(m) \rightarrow \mathcal{A}(m)$ by

$$
\mathcal{N}_{n, m, q}^{\lambda, \alpha} f(z) * \mathcal{M}_{q, \lambda+1}(z)=z \partial_{q} \mathcal{K}_{n, m}^{\alpha} f(z)
$$

where

$$
\mathcal{M}_{q, \lambda+1}(z):=z+\sum_{k=m+1}^{\infty} \frac{[\lambda+1, q]_{k-1}}{[k-1, q] !} z^{k}, z \in \mathbb{U} .
$$

From the above definition, we obtain

$$
\begin{gathered}
\mathcal{N}_{n, m, q}^{\lambda, \alpha} f(z)=z+\sum_{k=m+1}^{\infty}\left(\frac{n+1}{n+k}\right)^{\alpha} \frac{[k, q][k-1, q] !}{[\lambda+1, q]_{k-1}} a_{k} z^{k} \\
=z+\sum_{k=m+1}^{\infty} \frac{[k, q] !}{[\lambda+1, q]_{k-1}}\left(\frac{n+1}{n+k}\right)^{\alpha} a_{k} z^{k}, z \in \mathbb{U}, \\
(\alpha>0, \lambda>-1, m \geq 0,0<q<1)
\end{gathered}
$$

and from Equation (3) we can easily verify that

$$
[\lambda+1, q] \mathcal{N}_{n, m, q}^{\lambda, \alpha} f(z)=[\lambda, q] \mathcal{N}_{n, m, q}^{\lambda+1, \alpha} f(z)+q^{\lambda} z \partial_{q} \mathcal{N}_{n, m, q}^{\lambda+1, \alpha} f(z), z \in \mathbb{U} .
$$


We note that

$$
\lim _{q \rightarrow 1^{-}} \mathcal{N}_{n, m, q}^{\lambda, \alpha} f(z)=: \mathcal{I}_{n, m}^{\lambda, \alpha} f(z)=z+\sum_{k=m+1}^{\infty} \frac{k !}{(\lambda+1)_{k-1}}\left(\frac{n+1}{n+k}\right)^{\alpha} a_{k} z^{k}, z \in \mathbb{U} .
$$

Definition 1. For $f, g \in \mathcal{H}(\mathbb{U})$, we say that $f$ is subordinate to $g$, written $f(z) \prec g(z)$, if there exists a Schwarz function $w$, which is analytic in $\mathbb{U}$, with $w(0)=0$ and $|w(z)|<1$ for all $z \in \mathbb{U}$, such that $f(z)=g(w(z)), z \in \mathbb{U}$. Furthermore, if the function $g$ is univalent in $\mathbb{U}$, then we have the following equivalence $($ see $[4,5])$ :

$$
f(z) \prec g(z) \Leftrightarrow f(0)=g(0) \text { and } f(\mathbb{U}) \subset g(\mathbb{U})
$$

Let $k, h \in \mathcal{H}(\mathbb{U})$, and let $\varphi(r, s ; z): \mathbb{C}^{2} \times \mathbb{U} \rightarrow \mathbb{C}$.

(i) If $k$ satisfies the first order differential subordination

$$
\varphi\left(k(z), z k^{\prime}(z) ; z\right) \prec h(z),
$$

then $k$ is said to be a solution of the differential subordination in Equation (5). The function $q$ is called $a$ dominant of the solutions of the differential subordination in Equation (5) if $k(z) \prec q(z)$ for all the functions $k$ satisfying Equation (5). A dominant $\widetilde{q}$ is said to be the best dominant of Equation (5) if $\widetilde{q}(z) \prec q(z)$ for all the dominants $q$.

(ii) If $k$ satisfies the first order differential superordination

$$
h(z) \prec \varphi\left(k(z), z k^{\prime}(z) ; z\right),
$$

then $k$ is called to be $a$ solution of the differential superordination in Equation (6). The function $q$ is called a subordinant of the solutions of the differential superordination in Equation (6) if $q(z) \prec k(z)$ for all the functions $k$ satisfying Equation (6). A subordinant $\widetilde{q}$ is said to be the best subordinant of Equation (6) if $q(z) \prec \widetilde{q}(z)$ for all the subordinants $q$.

Miller and Mocanu [6] obtained conditions on the functions $h, q$ and $\varphi$ for which the following implication holds:

$$
h(z) \prec \varphi\left(k(z), z k^{\prime}(z) ; z\right) \Rightarrow q(z) \prec k(z) .
$$

Applying these methods, in $[7,8]$, the author studied general classes of first order differential superordinations and superordination-preserving integral operators. Using the results of Bulboacă [4] (see also [9,10]), the authors of [11] obtained sufficient conditions for functions $f \in \mathcal{A}$ to satisfy the double subordination

$$
q_{1}(z) \prec \frac{z f^{\prime}(z)}{f(z)} \prec q_{2}(z),
$$

where $q_{1}$ and $q_{2}$ are univalent functions in $\mathbb{U}$, normalized with $q_{1}(0)=q_{2}(0)=1$.

Sakaguchi [12] introduced a class $S_{s}^{*}$ of functions starlike with respect to symmetric points, which consists of functions $f \in \mathcal{A}$ satisfying the inequality

$$
\operatorname{Re} \frac{z f^{\prime}(z)}{f(z)-f(-z)}>0, z \in \mathbb{U}
$$

that represents a subclass of close-to-convex functions, and hence univalent in $\mathbb{U}$. Moreover, this class includes the class of convex functions and odd starlike functions with respect to the origin (see [12,13]).

In addition, Aouf et al. [14] introduced and studied the class $S_{s, n}^{*} T(1,1)$ of functions n-starlike with respect to symmetric points, which consists of functions $f \in \mathcal{A}$, with $a_{k} \leq 0$ for $k \geq 2$, and satisfying the inequality

$$
\operatorname{Re} \frac{D^{n+1} f(z)}{D^{n} f(z)-D^{n} f(-z)}>0, z \in \mathbb{U}
$$


where $D^{n}$ is the Sălăgean operator [15].

The classes defined in $[12,13]$ could be generalized by introducing the next class of functions, defined with the aid of the $\mathcal{N}_{n, m, q}^{\lambda, \alpha}$ operator defined as follows:

Definition 2. A function $f \in \mathcal{A}(m)$ with

$$
\mathcal{N}_{n, m, q}^{\lambda, \alpha} f(z)-\mathcal{N}_{n, m, q}^{\lambda, \alpha} f(-z) \neq 0, z \in \mathbb{U}:=\mathbb{U} \backslash\{0\},
$$

is said to be in the class $\mathcal{M}_{n, m, q}^{\lambda, \alpha}(\gamma, \mu, A, B)$ if it satisfies the subordination condition

$$
\begin{gathered}
(1+\gamma)\left(\frac{2 z}{\mathcal{N}_{n, m, q}^{\lambda, \alpha} f(z)-\mathcal{N}_{n, m, q}^{\lambda, \alpha} f(-z)}\right)^{\mu} \\
-\gamma\left(\frac{z\left(\mathcal{N}_{n, m, q}^{\lambda, \alpha} f(z)\right)^{\prime}-z\left(\mathcal{N}_{n, m, q}^{\lambda, q} f(-z)\right)^{\prime}}{\mathcal{N}_{n, m, q}^{\lambda, \alpha} f(z)-\mathcal{N}_{n, m, q}^{\lambda, \alpha} f(-z)}\right)\left(\frac{2 z}{\mathcal{N}_{n, m, q}^{\lambda, \alpha} f(z)-\mathcal{N}_{n, m, q}^{\lambda, \alpha} f(-z)}\right)^{\mu} \prec \frac{1+A z}{1+B z}, \\
(\gamma \in \mathbb{C}, 0<\mu<1,-1 \leq B<A \leq 1, m \in \mathbb{N}, \alpha>0, n \geq 0,0<q<1, \lambda>-1) .
\end{gathered}
$$

By specializing the parameters $\alpha, \lambda$ and $q$, we obtain the following subclasses:

(i) For $q \rightarrow 1^{-}$, we define the class $\mathcal{W}_{n, m}^{\lambda, \alpha}(\gamma, \mu, A, B)$ as follows:

$$
\begin{gathered}
\mathcal{W}_{n, m}^{\lambda, \alpha}(\gamma, \mu, A, B):=\left\{f \in \mathcal{A}(m):(1+\gamma)\left(\frac{2 z}{\mathcal{I}_{n, m}^{\lambda, \alpha} f(z)-\mathcal{I}_{n, m}^{\lambda, \alpha} f(-z)}\right)^{\mu}\right. \\
\left.-\gamma\left(\frac{z\left(\mathcal{I}_{n, m}^{\lambda, \alpha} f(z)\right)^{\prime}-z\left(\mathcal{I}_{n, m}^{\lambda, \alpha} f(-z)\right)^{\prime}}{\mathcal{I}_{n, m}^{\lambda, \alpha} f(z)-\mathcal{I}_{n, m}^{\lambda, \alpha} f(-z)}\right)\left(\frac{2 z}{\mathcal{I}_{n, m}^{\lambda, \alpha} f(z)-\mathcal{I}_{n, m}^{\lambda, \alpha} f(-z)}\right)^{\mu} \prec \frac{1+A z}{1+B z}\right\},
\end{gathered}
$$

where the operator $\mathcal{I}_{n, m}^{\lambda, \alpha}$ is defined by Equation (4);

(ii) For $q \rightarrow 1^{-}, \alpha=0$ and $\lambda=1$, we define the class $\mathcal{N}^{\gamma, \mu}(m, A, B)$ that corrects the class defined by Muhammad and Marwan [16] as follows:

$$
\begin{aligned}
& \mathcal{N}^{\gamma, \mu}(m, A, B):=\left\{f \in \mathcal{A}(m):(1+\gamma)\left(\frac{2 z}{f(z)-f(-z)}\right)^{\mu}\right. \\
& \left.-\gamma\left(\frac{z\left(f^{\prime}(z)-f^{\prime}(-z)\right)}{f(z)-f(-z)}\right)\left(\frac{2 z}{f(z)-f(-z)}\right)^{\mu} \prec \frac{1+A z}{1+B z}\right\} .
\end{aligned}
$$

In this paper, we obtain some sharp differential subordination and superordination results for the functions belonging to the class $\mathcal{M}_{n, m, q}^{\lambda, \alpha}(\gamma, \mu, A, B)$ to try to make a connection between a special subclass of analytic functions whose coefficients are given by the $q$-analog of integral operator and the differential subordination theory.

\section{Preliminaries}

To prove our results, we need the following definition and lemmas.

Definition 3 ([5]). (Definition 2.2b., p. 21) Let $\mathcal{Q}$ be the set of all functions $f$ that are analytic and injective on $\overline{\mathbb{U}} \backslash E(f)$, where $E(f):=\left\{\zeta \in \partial \mathbb{U}: \lim _{z \rightarrow \zeta} f(z)=\infty\right\}$ and are such that $f^{\prime}(\zeta) \neq 0$ for $\zeta \in \partial \mathbb{U} \backslash E(f)$.

Lemma 1 ([5]). (Theorem 3.1b., $p$. 71) Let the function $H$ be convex in $\mathbb{U}$, with $H(0)=a$, and $\zeta \neq 0$ with $\operatorname{Re} \zeta \geq 0$. If $\Phi \in \mathcal{H}[a, m]$ and

$$
\Phi(z)+\frac{z \Phi^{\prime}(z)}{\zeta} \prec H(z)
$$


then

$$
\Phi(z) \prec \Psi(z):=\frac{\zeta}{m z^{\frac{\zeta}{m}}} \int_{0}^{z} t^{\frac{\zeta}{m}-1} H(t) d t \prec H(z),
$$

and the function $\Psi$ is convex, $\Psi \in \mathcal{H}[a, m]$, and is the best dominant of Equation (9).

Lemma 2 ([17]). (Lemma 2.2., $p$. 3) Let $q$ be univalent in $\mathbb{U}$, with $q(0)=1$. Let $\xi, \varphi \in \mathbb{C}$ with $\varphi \neq 0$, and assume that

$$
\operatorname{Re}\left(1+\frac{z q^{\prime \prime}(z)}{q^{\prime}(z)}\right)>\max \left\{0 ;-\operatorname{Re} \frac{\xi}{\varphi}\right\}, z \in \mathbb{U} .
$$

If $k$ is analytic in $\mathbb{U}$ and

$$
\xi k(z)+\varphi z k^{\prime}(z) \prec \xi q(z)+\varphi z q^{\prime}(z)
$$

then $k(z) \prec q(z)$, and $q$ is the best dominant of Equation (10).

From [6] (Theorem 6, p. 820), we could easily obtain the following lemma:

Lemma 3. Let $q$ be convex in $\mathbb{U}$, and $k \neq 0$ with $\operatorname{Re} k \geq 0$. If $g \in \mathcal{H}[q(0), 1] \cap \mathcal{Q}$, such that $g(z)+k z g^{\prime}(z)$ is univalent in $\mathbb{U}$, then

$$
q(z)+k z q^{\prime}(z) \prec g(z)+k z g^{\prime}(z),
$$

implies that $q(z) \prec g(z)$, and $q$ is the best subordinant of Equation (11).

Lemma 4 ([18]). Let $F$ be analytic and convex in $\mathbb{U}$, and $0 \leq \lambda \leq 1$. If $f, g \in \mathcal{A}$, such that $f(z) \prec F(z)$ and $g(z) \prec F(z)$, then

$$
\lambda f(z)+(1-\lambda) g(z) \prec F(z)
$$

\section{Main Results}

Unless otherwise mentioned, we assume in the remainder of this paper that $\gamma \in \mathbb{C}, 0<\mu<1$, $-1 \leq B<A \leq 1, m \in \mathbb{N}, \alpha>0, n \geq 0,0<q<1, \lambda>-1$, and all the powers are understood as principle values.

Theorem 1. If $f \in \mathcal{M}_{n, m, q}^{\lambda, \alpha}(\gamma, \mu, A, B)$ and $\gamma \in \mathbb{C}^{*}:=\mathbb{C} \backslash\{0\}$ with $\operatorname{Re} \gamma \geq 0$, then

$$
\left(\frac{2 z}{\mathcal{N}_{n, m, q}^{\lambda, \alpha} f(z)-\mathcal{N}_{n, m, q}^{\lambda, \alpha} f(-z)}\right)^{\mu} \prec \Psi(z):=\frac{\mu}{\gamma m} \int_{0}^{1} \frac{1+A z u}{1+B z u} u^{\frac{\mu}{\gamma m}-1} d u \prec \frac{1+A z}{1+B z},
$$

and $\Psi$ is convex, $\Psi \in \mathcal{H}[1, m]$, and is the best dominant.

Proof. If we define the function $h$ by

$$
h(z):=\left(\frac{2 z}{\mathcal{N}_{n, m, q}^{\lambda, \alpha} f(z)-\mathcal{N}_{n, m, q}^{\lambda, \alpha} f(-z)}\right)^{\mu}, z \in \mathbb{U},
$$

from Equation (7), it follows that $h$ is an analytic function in $\mathbb{U}$, with $h(0)=1$. Differentiating Equation (12) with respect to $z$, we obtain that 


$$
\begin{gathered}
(1+\gamma)\left(\frac{2 z}{\mathcal{N}_{n, m, q}^{\lambda, \alpha} f(z)-\mathcal{N}_{n, m, q}^{\lambda, \alpha} f(-z)}\right)^{\mu} \\
-\gamma\left(\frac{z\left(\mathcal{N}_{n, m, q}^{\lambda, \alpha} f(z)\right)^{\prime}-z\left(\mathcal{N}_{n, m, q}^{\lambda, \alpha} f(-z)\right)^{\prime}}{\mathcal{N}_{n, m, q}^{\lambda, \alpha} f(z)-\mathcal{N}_{n, m, q}^{\lambda, \alpha} f(-z)}\right)\left(\frac{2 z}{\mathcal{N}_{n, m, q}^{\lambda, \alpha} f(z)-\mathcal{N}_{n, m, q}^{\lambda, \alpha} f(-z)}\right)^{\mu} \\
=h(z)+\frac{\gamma}{\mu} z h^{\prime}(z) \prec \frac{1+A z}{1+B z} .
\end{gathered}
$$

Since

$$
\mathcal{N}_{n, m, q}^{\lambda, \alpha} f(z)=z+\sum_{k=m+1}^{\infty} \alpha_{k} z^{k}, \quad \text { and } \quad \mathcal{N}_{n, m, q}^{\lambda, \alpha} f(-z)=-z+\sum_{k=m+1}^{\infty} \alpha_{k}(-1)^{k} z^{k}
$$

where

$$
\alpha_{k}=\frac{[k, q] !}{[\lambda+1, q]_{k-1}}\left(\frac{n+1}{n+k}\right)^{\alpha} a_{k}, k \geq m+1
$$

we have

$$
U(z):=\frac{2 z}{\mathcal{N}_{n, m, q}^{\lambda, \alpha} f(z)-\mathcal{N}_{n, m, q}^{\lambda, \alpha} f(-z)}=\frac{2 z}{2 z+\sum_{k=m+1}^{\infty} \alpha_{k}\left[1+(-1)^{k+1}\right] z^{k}}=\frac{1}{1+\sum_{s=m}^{\infty} \beta_{s} z^{s}},
$$

with

$$
\beta_{s}=\frac{\alpha_{s+1}\left[1+(-1)^{s}\right]}{2}, s \geq m
$$

Moreover,

$$
U(z)=\frac{1}{1+\sum_{s=m}^{\infty} \beta_{s} z^{s}}=1+\sum_{j=1}^{\infty} \gamma_{j} z^{j}, z \in \mathbb{U}
$$

with unknowns $\gamma_{j}, j \geq 1$, we have

$$
1=\left(1+\beta_{m} z^{m}+\beta_{m+1} z^{m+1}+\ldots\right)\left(1+\gamma_{1} z+\gamma_{2} z^{2}+\cdots+\gamma_{m} z^{m}+\gamma_{m+1} z^{m+1}+\ldots\right),
$$

and equating the corresponding coefficients it follows that

$$
\gamma_{1}=\gamma_{2}=\cdots=\gamma_{m-1}=0, \quad \gamma_{m}=-\beta_{m}, \quad \gamma_{m+1}=-\beta_{m+1}, \ldots
$$

hence

$$
U(z)=1+\sum_{j=m}^{\infty} \gamma_{j} z^{j} \in \mathcal{H}[1, m] .
$$

According to Equation (12), we have

$$
h=U^{\mu}, \quad \text { with } \quad U \in \mathcal{H}[1, m],
$$

and using the binomial power expansion formula, we get

$$
h=U^{\mu} \in \mathcal{H}[1, m] .
$$

Now, from the subordination in Equation (13), using Lemma 1 for $\zeta=\frac{\mu}{\gamma}$, we obtain our result.

Taking $q \rightarrow 1^{-}$in Theorem 1 , we obtain the following corollary: 
Corollary 1. If $f \in \mathcal{W}_{n, m}^{\lambda, \alpha}(\gamma, \mu, A, B)$ and $\gamma \in \mathbb{C}^{*}:=\mathbb{C} \backslash\{0\}$ with $\operatorname{Re} \gamma \geq 0$, then

$$
\left(\frac{2 z}{\mathcal{I}_{n, m}^{\lambda, \alpha} f(z)-\mathcal{I}_{n, m}^{\lambda, \alpha} f(-z)}\right)^{\mu} \prec \Psi(z):=\frac{\mu}{\gamma m} \int_{0}^{1} \frac{1+A z u}{1+B z u} u^{\frac{\mu}{\gamma m}-1} d u \prec \frac{1+A z}{1+B z},
$$

and $\Psi$ is convex, $\Psi \in \mathcal{H}[1, m]$, and is the best dominant.

Remark 1. The above theorem shows that

$$
\mathcal{M}_{n, m, q}^{\lambda, \alpha}(\gamma, \mu, A, B) \subset \mathcal{M}_{n, m, q}^{\lambda, \alpha}(0, \mu, A, B)
$$

for all $\gamma \in \mathbb{C}$ with $\operatorname{Re} \gamma \geq 0$.

Moreover, the next inclusion result for the classes $\mathcal{M}_{n, m, q}^{\lambda, \alpha}(\gamma, \mu, A, B)$ holds:

Theorem 2. If $\gamma_{1}, \gamma_{2} \in \mathbb{R}$ such that $0 \leq \gamma_{1} \leq \gamma_{2}$, and $-1 \leq B_{1} \leq B_{2}<A_{2} \leq A_{1} \leq 1$, then

$$
\mathcal{M}_{n, m, q}^{\lambda, \alpha}\left(\gamma_{2}, \mu, A_{2}, B_{2}\right) \subset \mathcal{M}_{n, m, q}^{\lambda, \alpha}\left(\gamma_{1}, \mu, A_{1}, B_{1}\right)
$$

Proof. If $f \in \mathcal{M}_{n, m, q}^{\lambda, \alpha}\left(\gamma_{2}, \mu, A_{2}, B_{2}\right)$, since $-1 \leq B_{1} \leq B_{2}<A_{2} \leq A_{1} \leq 1$, it is easy to check that

$$
\begin{gathered}
\left(1+\gamma_{2}\right)\left(\frac{2 z}{\mathcal{N}_{n, m, q}^{\lambda, \alpha} f(z)-\mathcal{N}_{n, m, q}^{\lambda, \alpha} f(-z)}\right)^{\mu} \\
-\gamma_{2}\left(\frac{z\left(\mathcal{N}_{n, m, q}^{\lambda, \alpha} f(z)-\mathcal{N}_{n, m, q}^{\lambda, \alpha} f(-z)\right)^{\prime}}{\mathcal{N}_{n, m, q}^{\lambda, \alpha} f(z)-\mathcal{N}_{n, m, q}^{\lambda, \alpha} f(-z)}\right)\left(\frac{2 z}{\mathcal{N}_{n, m, q}^{\lambda, \alpha} f(z)-\mathcal{N}_{n, m, q}^{\lambda, \alpha} f(-z)}\right)^{\mu} \\
\prec \frac{1+A_{2} z}{1+B_{2} z} \prec \frac{1+A_{1} z}{1+B_{1} z},
\end{gathered}
$$

that is $f \in \mathcal{M}_{n, m, q}^{\lambda, \alpha}\left(\gamma_{1}, \mu, A_{1}, B_{1}\right)$, hence the assertion in Equation (14) holds for $\gamma_{1}=\gamma_{2}$.

If $0 \leq \gamma_{1}<\gamma_{2}$, from Remark 1 and Equation (15), it follows $f \in \mathcal{M}_{n, m, q}^{\lambda, \alpha}\left(0, \mu, A_{1}, B_{1}\right)$, that is

$$
\left(\frac{2 z}{\mathcal{N}_{n, m, q}^{\lambda, \alpha} f(z)-\mathcal{N}_{n, m, q}^{\lambda, \alpha} f(-z)}\right)^{\mu} \prec \frac{1+A_{1} z}{1+B_{1} z} .
$$

A simple computation shows that

$$
\begin{aligned}
& \left(1+\gamma_{1}\right)\left(\frac{2 z}{\mathcal{N}_{n, m, q}^{\lambda, \alpha} f(z)-\mathcal{N}_{n, m, q}^{\lambda, \alpha} f(-z)}\right)^{\mu} \\
& -\gamma_{1}\left(\frac{z\left(\mathcal{N}_{n, m, q}^{\lambda, \alpha} f(z)-\mathcal{N}_{n, m, q}^{\lambda, \alpha} f(-z)\right)^{\prime}}{\mathcal{N}_{n, m, q}^{\lambda, \alpha} f(z)-\mathcal{N}_{n, m, q}^{\lambda, \alpha} f(-z)}\right)\left(\frac{2 z}{\mathcal{N}_{n, m, q}^{\lambda, \alpha} f(z)-\mathcal{N}_{n, m, q}^{\lambda, \alpha} f(-z)}\right)^{\mu} \\
& =\left(1-\frac{\gamma_{1}}{\gamma_{2}}\right)\left(\frac{2 z}{\mathcal{N}_{n, m, q}^{\lambda, \alpha} f(z)-\mathcal{N}_{n, m, q}^{\lambda, \alpha} f(-z)}\right)^{\mu} \\
& +\frac{\gamma_{1}}{\gamma_{2}}\left[\left(1+\gamma_{2}\right)\left(\frac{2 z}{\mathcal{N}_{n, m, q}^{\lambda, \alpha} f(z)-\mathcal{N}_{n, m, q}^{\lambda, \alpha} f(-z)}\right)^{\mu}\right. \\
& \left.-\gamma_{2}\left(\frac{z\left(\mathcal{N}_{n, m, q}^{\lambda, \alpha} f(z)-\mathcal{N}_{n, m, q}^{\lambda, \alpha} f(-z)\right)^{\prime}}{\mathcal{N}_{n, m, q}^{\lambda, \alpha} f(z)-\mathcal{N}_{n, m, q}^{\lambda, \alpha} f(-z)}\right)\left(\frac{2 z}{\mathcal{N}_{n, m, q}^{\lambda, \alpha} f(z)-\mathcal{N}_{n, m, q}^{\lambda, \alpha} f(-z)}\right)^{\mu}\right], z \in \mathbb{U} .
\end{aligned}
$$

Moreover,

$$
0 \leq \frac{\gamma_{1}}{\gamma_{2}}<1
$$


and the function $\frac{1+A_{1} z}{1+B_{1} z}$, with $-1 \leq B_{1}<A_{1} \leq 1$, is analytic and convex in $\mathbb{U}$. According to Equation (17), using the subordinations in Equations (15) and (16), from Lemma 4, we deduce that

$$
\begin{gathered}
\left(1+\gamma_{1}\right)\left(\frac{2 z}{\mathcal{N}_{n, m, q}^{\lambda, \alpha} f(z)-\mathcal{N}_{n, m, q}^{\lambda, \alpha} f(-z)}\right)^{\mu} \\
-\gamma_{1}\left(\frac{z\left(\mathcal{N}_{n, m, q}^{\lambda, \alpha} f(z)-\mathcal{N}_{n, m, q}^{\lambda, \alpha} f(-z)\right)^{\prime}}{\mathcal{N}_{n, m, q}^{\lambda, \alpha} f(z)-\mathcal{N}_{n, m, q}^{\lambda, \alpha} f(-z)}\right)\left(\frac{2 z}{\mathcal{N}_{n, m, q}^{\lambda, \alpha} f(z)-\mathcal{N}_{n, m, q}^{\lambda, \alpha} f(-z)}\right)^{\mu} \prec \frac{1+A_{1} z}{1+B_{1} z},
\end{gathered}
$$

that is $f \in \mathcal{M}_{n, m, q}^{\lambda, \alpha}\left(\gamma_{1}, \mu, A_{1}, B_{1}\right)$.

Taking $q \rightarrow 1^{-}$in Theorem 2, we obtain the following corollary:

Corollary 2. If $\gamma_{1}, \gamma_{2} \in \mathbb{R}$ such that $0 \leq \gamma_{1} \leq \gamma_{2}$, and $-1 \leq B_{1} \leq B_{2}<A_{2} \leq A_{1} \leq 1$, then

$$
\mathcal{W}_{n, m}^{\lambda, \alpha}\left(\gamma_{2}, \mu, A_{2}, B_{2}\right) \subset \mathcal{W}_{n, m}^{\lambda, \alpha}\left(\gamma_{1}, \mu, A_{1}, B_{1}\right) .
$$

Example 1. For the special case $A_{1}=1$ and $B_{1}=-1$, Theorem 2 and Corollary 2 reduce to the next examples, respectively:

Suppose that $\gamma_{1}, \gamma_{2} \in \mathbb{R}$ such that $0 \leq \gamma_{1} \leq \gamma_{2}$, and $-1 \leq B_{2}<A_{2} \leq 1$.

1. If $f \in \mathcal{M}_{n, m, q}^{\lambda, \alpha}\left(\gamma_{2}, \mu, A_{2}, B_{2}\right)$, then

$$
\begin{gathered}
\operatorname{Re}\left\{\left(1+\gamma_{1}\right)\left(\frac{2 z}{\mathcal{N}_{n, m, q}^{\lambda, \alpha} f(z)-\mathcal{N}_{n, m, q}^{\lambda, \alpha} f(-z)}\right)^{\mu}\right. \\
\left.-\gamma_{1}\left(\frac{z\left(\mathcal{N}_{n, m, q}^{\lambda, \alpha} f(z)-\mathcal{N}_{n, m, q}^{\lambda, \alpha} f(-z)\right)^{\prime}}{\mathcal{N}_{n, m, q}^{\lambda, \alpha} f(z)-\mathcal{N}_{n, m, q}^{\lambda, \alpha} f(-z)}\right)\left(\frac{2 z}{\mathcal{N}_{n, m, q}^{\lambda, \alpha} f(z)-\mathcal{N}_{n, m, q}^{\lambda, \alpha} f(-z)}\right)^{\mu}\right\}>0, z \in \mathbb{U} ;
\end{gathered}
$$

2. If $f \in \mathcal{W}_{n, m}^{\lambda, \alpha}\left(\gamma_{2}, \mu, A_{2}, B_{2}\right)$, then

$$
\begin{gathered}
\operatorname{Re}\left\{\left(1+\gamma_{1}\right)\left(\frac{2 z}{\mathcal{I}_{n, m, q}^{\lambda, \alpha} f(z)-\mathcal{I}_{n, m, q}^{\lambda, \alpha} f(-z)}\right)^{\mu}\right. \\
\left.-\gamma_{1}\left(\frac{z\left(\mathcal{I}_{n, m, q}^{\lambda, \alpha} f(z)-\mathcal{I}_{n, m, q}^{\lambda, \alpha} f(-z)\right)^{\prime}}{\mathcal{I}_{n, m, q}^{\lambda, \alpha} f(z)-\mathcal{I}_{n, m, q}^{\lambda, \alpha} f(-z)}\right)\left(\frac{2 z}{\mathcal{I}_{n, m, q}^{\lambda, \alpha} f(z)-\mathcal{I}_{n, m, q}^{\lambda, \alpha} f(-z)}\right)^{\mu}\right\}>0, z \in \mathbb{U} ;
\end{gathered}
$$

Theorem 3. Suppose that $q$ is univalent in $\mathbb{U}$, with $q(0)=1$, and let $\gamma \in \mathbb{C}^{*}$ such that

$$
\operatorname{Re}\left(1+\frac{z q^{\prime \prime}(z)}{q^{\prime}(z)}\right)>\max \left\{0 ;-\operatorname{Re} \frac{\mu}{\gamma}\right\}, z \in \mathbb{U} .
$$

If $f \in \mathcal{A}(m)$ such that Equation (7) holds, and satisfies the subordination

$$
\begin{gathered}
(1+\gamma)\left(\frac{2 z}{\mathcal{N}_{n, m, q}^{\lambda, \alpha} f(z)-\mathcal{N}_{n, m, q}^{\lambda, \alpha} f(-z)}\right)^{\mu} \\
-\gamma\left(\frac{z\left(\mathcal{N}_{n, m, q}^{\lambda, \alpha} f(z)-\mathcal{N}_{n, m, q}^{\lambda, \alpha} f(-z)\right)^{\prime}}{\mathcal{N}_{n, m, q}^{\lambda, \alpha} f(z)-\mathcal{N}_{n, m, q}^{\lambda, \alpha} f(-z)}\right)\left(\frac{2 z}{\mathcal{N}_{n, m, q}^{\lambda, \alpha} f(z)-\mathcal{N}_{n, m, q}^{\lambda, \alpha} f(-z)}\right)^{\mu} \\
\prec q(z)+\frac{\gamma}{\mu} z q^{\prime}(z),
\end{gathered}
$$


then

$$
\left(\frac{2 z}{\mathcal{N}_{n, m, q}^{\lambda, \alpha} f(z)-\mathcal{N}_{n, m, q}^{\lambda, \alpha} f(-z)}\right)^{\mu} \prec q(z),
$$

and $q$ is the best dominant of Equation (19).

Proof. Since $f \in \mathcal{A}(m)$ such that Equation (7) holds, it follows that the function $h$ defined by Equation (12) is analytic in $\mathbb{U}$, and $h(0)=1$. As in the proof of Theorem 1, differentiating Equation (12) with respect to $z$, we obtain that Equation (19) is equivalent to

$$
h(z)+\frac{\gamma}{\mu} z h^{\prime}(z) \prec q(z)+\frac{\gamma}{\mu} z q^{\prime}(z) .
$$

Using Lemma 2 for $\xi:=1$ and $\varphi:=\frac{\gamma}{\mu}$, we get that the above subordination implies $h(z) \prec q(z)$, and $q$ is the best dominant of Equation (19).

For the special case $q(z)=\frac{1+A z}{1+B z}$, with $-1 \leq B<A \leq 1$, Theorem 3 reduces to the following corollary:

Corollary 3. Let $\gamma \in \mathbb{C}^{*}$ and $-1 \leq B<A \leq 1$, such that

$$
\max \left\{-1 ;-\frac{1+\operatorname{Re} \frac{\mu}{\gamma}}{1-\operatorname{Re} \frac{\mu}{\gamma}}\right\} \leq B \leq 0, \quad \text { or } \quad 0 \leq B \leq \min \left\{1 ; \frac{1+\operatorname{Re} \frac{\mu}{\gamma}}{1-\operatorname{Re} \frac{\mu}{\gamma}}\right\} .
$$

If $f \in \mathcal{A}(m)$ such that Equation (7) holds, and satisfies the subordination

$$
\begin{gathered}
(1+\gamma)\left(\frac{2 z}{\mathcal{N}_{n, m, q}^{\lambda, \alpha} f(z)-\mathcal{N}_{n, m, q}^{\lambda, \alpha} f(-z)}\right)^{\mu} \\
-\gamma\left(\frac{z\left(\mathcal{N}_{n, m, q}^{\lambda, \alpha} f(z)-\mathcal{N}_{n, m, q}^{\lambda, \alpha} f(-z)\right)^{\prime}}{\mathcal{N}_{n, m, q}^{\lambda, \alpha} f(z)-\mathcal{N}_{n, m, q}^{\lambda, \alpha} f(-z)}\right)\left(\frac{2 z}{\mathcal{N}_{n, m, q}^{\lambda, \alpha} f(z)-\mathcal{N}_{n, m, q}^{\lambda, \alpha} f(-z)}\right)^{\mu} \\
\prec \frac{1+A z}{1+B z}+\frac{\gamma}{\mu} \frac{(A-B) z}{(1+B z)^{2}},
\end{gathered}
$$

then

$$
\left(\frac{2 z}{\mathcal{N}_{n, m, q}^{\lambda, \alpha} f(z)-\mathcal{N}_{n, m, q}^{\lambda, \alpha} f(-z)}\right)^{\mu} \prec \frac{1+A z}{1+B z},
$$

and $\frac{1+A z}{1+B z}$ is the best dominant of Equation (21).

Proof. For $q(z)=\frac{1+A z}{1+B z}$, the condition in Equation (18) reduces to

$$
\operatorname{Re} \frac{1-B z}{1+B z}>\max \left\{0 ;-\operatorname{Re} \frac{\mu}{\gamma}\right\}, z \in \mathbb{U}
$$

Since

$$
\inf \left\{\operatorname{Re} \frac{1-B z}{1+B z}: z \in \mathbb{U}\right\}=\left\{\begin{array}{lll}
\frac{1+B}{1-B}, & \text { if } \quad-1 \leq B \leq 0 \\
\frac{1-B}{1+B}, & \text { if } \quad 0 \leq B<1
\end{array}\right.
$$

we easily check that Equation (22) holds if and only if the assumption in Equation (20) is satisfied, whenever $-1 \leq B<1$.

Taking $q \rightarrow 1^{-}$in Theorem 3, we obtain the following corollary: 
Corollary 4. Suppose that $q$ is univalent in $\mathbb{U}$, with $q(0)=1$, and let $\gamma \in \mathbb{C}^{*}$ such that

$$
\operatorname{Re}\left(1+\frac{z q^{\prime \prime}(z)}{q^{\prime}(z)}\right)>\max \left\{0 ;-\operatorname{Re} \frac{\mu}{\gamma}\right\}, z \in \mathbb{U} .
$$

If $f \in \mathcal{A}(m)$ such that Equation (7) holds, and satisfies the subordination

$$
\begin{gathered}
(1+\gamma)\left(\frac{2 z}{\mathcal{I}_{n, m}^{\lambda, \alpha} f(z)-\mathcal{I}_{n, m}^{\lambda, \alpha} f(-z)}\right)^{\mu} \\
-\gamma\left(\frac{z\left(\mathcal{I}_{n, m}^{\lambda, \alpha} f(z)-\mathcal{I}_{n, m}^{\lambda, \alpha} f(-z)\right)^{\prime}}{\mathcal{I}_{n, m}^{\lambda, \alpha} f(z)-\mathcal{I}_{n, m}^{\lambda, \alpha} f(-z)}\right)\left(\frac{2 z}{\mathcal{I}_{n, m}^{\lambda, \alpha} f(z)-\mathcal{I}_{n, m}^{\lambda, \alpha} f(-z)}\right)^{\mu} \prec q(z)+\frac{\gamma}{\mu} z q^{\prime}(z),
\end{gathered}
$$

then

$$
\left(\frac{2 z}{\mathcal{I}_{n, m}^{\lambda, \alpha} f(z)-\mathcal{I}_{n, m}^{\lambda, \alpha} f(-z)}\right)^{\mu} \prec q(z),
$$

and $q$ is the best dominant of Equation (19).

Theorem 4. Let $q$ be convex in $\mathbb{U}$, with $q(0)=1$, and $\gamma \in \mathbb{C}^{*}$, with $\operatorname{Re} \gamma \geq 0$. In addition, let $f \in \mathcal{A}(m)$ such that

$$
\left(\frac{2 z}{\mathcal{N}_{n, m, q}^{\lambda, \alpha} f(z)-\mathcal{N}_{n, m, q}^{\lambda, \alpha} f(-z)}\right)^{\mu} \in \mathcal{H}[q(0), 1] \cap \mathcal{Q},
$$

and assume that the function

$$
\begin{gathered}
(1+\gamma)\left(\frac{2 z}{\mathcal{N}_{n, m, q}^{\lambda, \alpha} f(z)-\mathcal{N}_{n, m, q}^{\lambda, \alpha} f(-z)}\right)^{\mu} \\
-\gamma\left(\frac{z\left(\mathcal{N}_{n, m, q}^{\lambda, \alpha} f(z)-\mathcal{N}_{n, m, q}^{\lambda, \alpha} f(-z)\right)^{\prime}}{\mathcal{N}_{n, m, q}^{\lambda, \alpha} f(z)-\mathcal{N}_{n, m, q}^{\lambda, \alpha} f(-z)}\right)\left(\frac{2 z}{\mathcal{N}_{n, m, q}^{\lambda, \alpha} f(z)-\mathcal{N}_{n, m, q}^{\lambda, \alpha} f(-z)}\right)^{\mu}
\end{gathered}
$$

is univalent in $\mathbb{U}$.

If

$$
\begin{gathered}
q(z)+\frac{\gamma}{\mu} z q^{\prime}(z) \prec(1+\gamma)\left(\frac{2 z}{\mathcal{N}_{n, m, q}^{\lambda, \alpha} f(z)-\mathcal{N}_{n, m, q}^{\lambda, \alpha} f(-z)}\right)^{\mu} \\
-\gamma\left(\frac{z\left(\mathcal{N}_{n, m, q}^{\lambda, \alpha} f(z)-\mathcal{N}_{n, m, q}^{\lambda, \alpha} f(-z)\right)^{\prime}}{\mathcal{N}_{n, m, q}^{\lambda, \alpha} f(z)-\mathcal{N}_{n, m, q}^{\lambda, \alpha} f(-z)}\right)\left(\frac{2 z}{\mathcal{N}_{n, m, q}^{\lambda, \alpha} f(z)-\mathcal{N}_{n, m, q}^{\lambda, \alpha} f(-z)}\right)^{\mu},
\end{gathered}
$$

then

$$
q(z) \prec\left(\frac{2 z}{\mathcal{N}_{n, m, q}^{\lambda, \alpha} f(z)-\mathcal{N}_{n, m, q}^{\lambda, \alpha} f(-z)}\right)^{\mu},
$$

and $q$ is the best subordinant of Equation (25).

Proof. Letting the function $h$ be defined by Equation (12), then $h \in \mathcal{H}[q(0), m]$, and from Equation (23) we have that $h \in \mathcal{H}[q(0), 1] \cap \mathcal{Q}$. As in the proof of Theorem 1, differentiating Equation (12) with respect to $z$, we obtain that

$$
q(z)+\frac{\gamma}{\mu} z q^{\prime}(z) \prec h(z)+\frac{\gamma}{\mu} z h^{\prime}(z)
$$

Now, according to Lemma 3 for $k:=\frac{\gamma}{\mu}$ we obtain the desired result.

Taking $q(z)=\frac{1+A z}{1+B z}$, with $-1 \leq B<A \leq 1$, in Theorem 4, we obtain the following corollary: 
Corollary 5. Let $\gamma \in \mathbb{C}^{*}$, with $\operatorname{Re} \gamma \geq 0$, and $-1 \leq B<A \leq 1$. If $f \in \mathcal{A}(m)$ such that the assumptions in Equations (23) and (24) hold, and satisfies the subordination

$$
\begin{gathered}
\frac{1+A z}{1+B z}+\frac{\gamma}{\mu} \frac{(A-B) z}{(1+B z)^{2}} \prec(1+\gamma)\left(\frac{2 z}{\mathcal{N}_{n, m, q}^{\lambda, \alpha} f(z)-\mathcal{N}_{n, m, q}^{\lambda, \alpha} f(-z)}\right)^{\mu} \\
-\gamma\left(\frac{z\left(\mathcal{N}_{n, m, q}^{\lambda, \alpha} f(z)-\mathcal{N}_{n, m, q}^{\lambda, \alpha} f(-z)\right)^{\prime}}{\mathcal{N}_{n, m, q}^{\lambda, \alpha} f(z)-\mathcal{N}_{n, m, q}^{\lambda, \alpha} f(-z)}\right)\left(\frac{2 z}{\mathcal{N}_{n, m, q}^{\lambda, \alpha} f(z)-\mathcal{N}_{n, m, q}^{\lambda, \alpha} f(-z)}\right)^{\mu},
\end{gathered}
$$

then

$$
\frac{1+A z}{1+B z} \prec\left(\frac{2 z}{\mathcal{N}_{n, m, q}^{\lambda, \alpha} f(z)-\mathcal{N}_{n, m, q}^{\lambda, \alpha} f(-z)}\right)^{\mu},
$$

and $\frac{1+A z}{1+B z}$ is the best subordinant of Equation (26).

Taking $q \rightarrow 1^{-}$in Theorem 4 , we obtain the following corollary:

Corollary 6. Let $q$ be convex in $\mathbb{U}$, with $q(0)=1$, and $\gamma \in \mathbb{C}^{*}$, with $\operatorname{Re} \gamma \geq 0$. In addition, let $f \in \mathcal{A}(m)$ such that

$$
\left(\frac{2 z}{\mathcal{I}_{n, m}^{\lambda, \alpha} f(z)-\mathcal{I}_{n, m}^{\lambda, \alpha} f(-z)}\right)^{\mu} \in \mathcal{H}[q(0), 1] \cap \mathcal{Q},
$$

and assume that the function

$$
\begin{gathered}
(1+\gamma)\left(\frac{2 z}{\mathcal{I}_{n, m}^{\lambda, \alpha} f(z)-\mathcal{I}_{n, m}^{\lambda, \alpha} f(-z)}\right)^{\mu} \\
-\gamma\left(\frac{z\left(\mathcal{I}_{n, m}^{\lambda, \alpha} f(z)-\mathcal{I}_{n, m}^{\lambda, \alpha} f(-z)\right)^{\prime}}{\mathcal{I}_{n, m}^{\lambda, \alpha} f(z)-\mathcal{I}_{n, m}^{\lambda, \alpha} f(-z)}\right)\left(\frac{2 z}{\mathcal{I}_{n, m}^{\lambda, \alpha} f(z)-\mathcal{I}_{n, m}^{\lambda, \alpha} f(-z)}\right)^{\mu} \text { is univalent in } \mathbb{U} .
\end{gathered}
$$

If

$$
\begin{gathered}
q(z)+\frac{\gamma}{\mu} z q^{\prime}(z) \prec(1+\gamma)\left(\frac{2 z}{\mathcal{I}_{n, m}^{\lambda, \alpha} f(z)-\mathcal{I}_{n, m}^{\lambda, \alpha} f(-z)}\right)^{\mu} \\
-\gamma\left(\frac{z\left(\mathcal{I}_{n, m}^{\lambda, \alpha} f(z)-\mathcal{I}_{n, m}^{\lambda, \alpha} f(-z)\right)^{\prime}}{\mathcal{I}_{n, m}^{\lambda, \alpha} f(z)-\mathcal{I}_{n, m}^{\lambda, \alpha} f(-z)}\right)\left(\frac{2 z}{\mathcal{I}_{n, m}^{\lambda, \alpha} f(z)-\mathcal{I}_{n, m}^{\lambda, \alpha} f(-z)}\right)^{\mu},
\end{gathered}
$$

then

$$
q(z) \prec\left(\frac{2 z}{\mathcal{I}_{n, m}^{\lambda, \alpha} f(z)-\mathcal{I}_{n, m}^{\lambda, \alpha} f(-z)}\right)^{\mu},
$$

and $q$ is the best subordinant of Equation (25).

Combining Theorems 3 and 4, we obtain the following sandwich-type theorem: 
Theorem 5. Let $q_{1}$ and $q_{2}$ be two convex functions in $\mathbb{U}$, with $q_{1}(0)=q_{2}(0)=1$, and let $\gamma \in \mathbb{C}^{*}$, with $\operatorname{Re} \gamma \geq 0$. If $f \in \mathcal{A}(m)$ such that the assumptions in Equations (23) and (24) hold, then

$$
\begin{gathered}
q_{1}(z)+\frac{\gamma}{\mu} z q_{1}^{\prime}(z) \prec \Theta(z):=(1+\gamma)\left(\frac{2 z}{\mathcal{N}_{n, m, q}^{\lambda, \alpha} f(z)-\mathcal{N}_{n, m, q}^{\lambda, \alpha} f(-z)}\right)^{\mu} \\
-\gamma\left(\frac{z\left(\mathcal{N}_{n, m, q}^{\lambda, \alpha} f(z)-\mathcal{N}_{n, m, q}^{\lambda, \alpha} f(-z)\right)^{\prime}}{\mathcal{N}_{n, m, n}^{\lambda, \alpha} f(z)-\mathcal{N}_{n, m, q}^{\lambda, \alpha} f(-z)}\right)\left(\frac{2 z}{\mathcal{N}_{n, m, q}^{\lambda, \alpha} f(z)-\mathcal{N}_{n, m, q}^{\lambda, \alpha} f(-z)}\right)^{\mu} \\
\prec q_{2}(z)+\frac{\gamma}{\mu} z q_{2}^{\prime}(z),
\end{gathered}
$$

implies that

$$
q_{1}(z) \prec \Phi(z):=\left(\frac{2 z}{\mathcal{N}_{n, m, q}^{\lambda, \alpha} f(z)-\mathcal{N}_{n, m, q}^{\lambda, \alpha} f(-z)}\right)^{\mu} \prec q_{2}(z),
$$

and $q_{1}$ and $q_{2}$ are, respectively, the best subordinant and the best dominant of Equation (27).

Combining Corollaries 4 and 6, we obtain the following sandwich-type theorem:

Corollary 7. Let $q_{1}$ and $q_{2}$ be two convex functions in $\mathbb{U}$, with $q_{1}(0)=q_{2}(0)=1$, and let $\gamma \in \mathbb{C}^{*}$, with $\operatorname{Re} \gamma \geq 0$. If $f \in \mathcal{A}(m)$ such that the assumptions in Equations (23) and (24) hold for the operator $\mathcal{N}_{n, m, q}^{\lambda, \alpha}$ replaced by $\mathcal{I}_{n, m, q}^{\lambda, \alpha}$, then

$$
\begin{gathered}
q_{1}(z)+\frac{\gamma}{\mu} z q_{1}^{\prime}(z) \prec \widehat{\Theta}(z):=(1+\gamma)\left(\frac{2 z}{\mathcal{I}_{n, m}^{\lambda, \alpha} f(z)-\mathcal{I}_{n, m}^{\lambda, \alpha} f(-z)}\right)^{\mu} \\
-\gamma\left(\frac{z\left(\mathcal{I}_{n, m}^{\lambda, \alpha} f(z)-\mathcal{I}_{n, m}^{\lambda, \alpha} f(-z)\right)^{\prime}}{\mathcal{I}_{n, m}^{\lambda, \alpha} f(z)-\mathcal{I}_{n, m}^{\lambda, \alpha} f(-z)}\right)\left(\frac{2 z}{\mathcal{I}_{n, m}^{\lambda, \alpha} f(z)-\mathcal{I}_{n, m}^{\lambda, \alpha} f(-z)}\right)^{\mu} \prec q_{2}(z)+\frac{\gamma}{\mu} z q_{2}^{\prime}(z),
\end{gathered}
$$

implies that

$$
q_{1}(z) \prec \widehat{\Phi}(z):=\left(\frac{2 z}{\mathcal{I}_{n, m}^{\lambda, \alpha} f(z)-\mathcal{I}_{n, m}^{\lambda, \alpha} f(-z)}\right)^{\mu} \prec q_{2}(z),
$$

and $q_{1}$ and $q_{2}$ are, respectively, the best subordinant and the best dominant of Equation (27).

Example 2. Taking $q_{j}=1+r_{j} z$, with $0<r_{1}<r_{2}, j=1,2$ in Theorem 5 and Corollary 7 , we obtain the next examples, respectively:

Let $\gamma \in \mathbb{C}^{*}$, with $\operatorname{Re} \gamma \geq 0$.

1. If $f \in \mathcal{A}(m)$ such that the assumptions in Equations (23) and (24) hold, then

$$
r_{1}\left|1+\frac{\gamma}{\mu}\right|<|\Theta(z)-1|<r_{2}\left|1+\frac{\gamma}{\mu}\right|, z \in \mathbb{U} \Rightarrow r_{1}<|\Phi(z)-1|<r_{2}, z \in \mathbb{U}, \quad\left(0<r_{1}<r_{2}\right)
$$

where $\Theta$ and $\Phi$ are given in Theorem 5, and the obtained bounds $r_{1}$ and $r_{2}$ are the best possible.

2. If $f \in \mathcal{A}(m)$ such that the assumptions in Equations (23) and (24) hold for the operator $\mathcal{N}_{n, m, q}^{\lambda, \alpha}$ replaced by $\mathcal{I}_{n, m, q}^{\lambda, \alpha}$, then

$$
r_{1}\left|1+\frac{\gamma}{\mu}\right|<|\widehat{\Theta}(z)-1|<r_{2}\left|1+\frac{\gamma}{\mu}\right|, z \in \mathbb{U} \Rightarrow r_{1}<|\widehat{\Phi}(z)-1|<r_{2}, z \in \mathbb{U}, \quad\left(0<r_{1}<r_{2}\right)
$$

where $\widehat{\Theta}$ and $\widehat{\Phi}$ are given in Corollary 7 , and the obtained bounds $r_{1}$ and $r_{2}$ are the best possible.

Example 3. Putting $q_{j}=e^{r_{j} z}$, with $0<r_{1}<r_{2} \leq 1, j=1,2$ in Theorem 5 and Corollary 7 , we obtain the next examples, respectively:

Let $\gamma \in \mathbb{C}^{*}$, with $\operatorname{Re} \gamma \geq 0$. 
1. If $f \in \mathcal{A}(m)$ such that the assumptions in Equations (23) and (24) hold, then

$$
\left(1+\frac{\gamma}{\mu} z\right) e^{r_{1} z} \prec \Theta(z) \prec\left(1+\frac{\gamma}{\mu} z\right) e^{r_{2} z} \Rightarrow e^{r_{1} z} \prec \Phi(z) \prec e^{r_{2} z}, \quad\left(0<r_{1}<r_{2} \leq 1\right)
$$

where $\Theta$ and $\Phi$ are given in Theorem 5 , and $e^{r_{1} z}$ and $e^{r_{2} z}$ are, respectively, the best subordinant and the best dominant.

2. If $f \in \mathcal{A}(m)$ such that the assumptions in Equations (23) and (24) hold for the operator $\mathcal{N}_{n, m, q}^{\lambda, \alpha}$ replaced by $\mathcal{I}_{n, m, q}^{\lambda, \alpha}$, then

$$
\left(1+\frac{\gamma}{\mu} z\right) e^{r_{1} z} \prec \widehat{\Theta}(z) \prec\left(1+\frac{\gamma}{\mu} z\right) e^{r_{2} z} \Rightarrow e^{r_{1} z} \prec \widehat{\Phi}(z) \prec e^{r_{2} z}, \quad\left(0<r_{1}<r_{2} \leq 1\right)
$$

where $\widehat{\Theta}$ and $\widehat{\Phi}$ are given in Corollary 7 , and $e^{r_{1} z}$ and $e^{r_{2} z}$ are, respectively, the best subordinant and the best dominant.

Theorem 6. If $f \in \mathcal{M}_{n, m, q}^{\lambda, \alpha}(0, \mu, 1-2 \rho,-1)$, with $0 \leq \rho<1$, then $f \in \mathcal{M}_{n, m, q}^{\lambda, \alpha}(\gamma, \mu, 1-2 \rho,-1)$ for $|z|<R$, where

$$
R=\left(\sqrt{\frac{|\gamma|^{2} m^{2}}{\mu^{2}}+1}-\frac{|\gamma| m}{\mu}\right)^{\frac{1}{m}} .
$$

Proof. For $f \in \mathcal{M}_{n, m, q}^{\lambda, \alpha}(0, \mu, 1-2 \rho,-1)$, with $0 \leq \rho<1$, let the function $h$ be defined by

$$
\left(\frac{2 z}{\mathcal{N}_{n, m, q}^{\lambda, \alpha} f(z)-\mathcal{N}_{n, m, q}^{\lambda, \alpha} f(-z)}\right)^{\mu}=(1-\rho) h(z)+\rho, z \in \mathbb{U} .
$$

Hence, the function $h$ is analytic in $\mathbb{U}$, with $h(0)=1$, and since $f \in \mathcal{M}_{n, m, q}^{\lambda, \alpha}(0, \mu, 1-2 \rho,-1)$ is equivalent to,

$$
\left(\frac{2 z}{\mathcal{N}_{n, m, q}^{\lambda, \alpha} f(z)-\mathcal{N}_{n, m, q}^{\lambda, \alpha} f(-z)}\right)^{\mu} \prec \frac{1+(1-2 \rho) z}{1-z},
$$

it follows that $\operatorname{Re} h(z)>0, z \in \mathbb{U}$.

As in the proof of Theorem 1 , since $f \in \mathcal{M}_{n, m, q}^{\lambda, \alpha}(0, \mu, 1-2 \rho,-1)$, with $0 \leq \rho<1$, we deduce that

$$
\left(\frac{2 z}{\mathcal{N}_{n, m, q}^{\lambda, \alpha} f(z)-\mathcal{N}_{n, m, q}^{\lambda, \alpha} f(-z)}\right)^{\mu} \in \mathcal{H}[1, m],
$$

and from the relation in Equation (30), we get $h \in \mathcal{H}[1, m]$. Therefore, the following estimate holds

$$
\left|z h^{\prime}(z)\right| \leq \frac{2 m r^{m} \operatorname{Re} h(z)}{1-r^{2 m}},|z|=r<1,
$$

that represents the result of Shah [19] (the inequality (6), p. 240, for $\alpha=0$ ), which generalize Lemma 2 of [20]. 
A simple computation shows that

$$
\begin{gathered}
\frac{1}{1-\rho}\left\{(1+\gamma)\left(\frac{2 z}{\mathcal{N}_{n, m, q}^{\lambda, \alpha} f(z)-\mathcal{N}_{n, m, q}^{\lambda, \alpha} f(-z)}\right)^{\mu}\right. \\
\left.-\gamma\left(\frac{z\left(\mathcal{N}_{n, m, q}^{\lambda, \alpha} f(z)-\mathcal{N}_{n, m, q}^{\lambda, \alpha} f(-z)\right)^{\prime}}{\mathcal{N}_{n, m, q}^{\lambda, \alpha} f(z)-\mathcal{N}_{n, m, q}^{\lambda, \alpha} f(-z)}\right)\left(\frac{2 z}{\mathcal{N}_{n, m, q}^{\lambda, \alpha} f(z)-\mathcal{N}_{n, m, q}^{\lambda, \alpha} f(-z)}\right)^{\mu}-\rho\right\} \\
=h(z)+\frac{\gamma}{\mu} z h^{\prime}(z), z \in \mathbb{U},
\end{gathered}
$$

hence, we obtain

$$
\begin{gathered}
\operatorname{Re}\left\{\frac { 1 } { 1 - \rho } \left[(1+\gamma)\left(\frac{2 z}{\mathcal{N}_{n, m, q}^{\lambda, \alpha} f(z)-\mathcal{N}_{n, m, q}^{\lambda, \alpha} f(-z)}\right)^{\mu}\right.\right. \\
\left.\left.-\gamma\left(\frac{z\left(\mathcal{N}_{n, m, q}^{\lambda, \alpha} f(z)-\mathcal{N}_{n, m, q}^{\lambda, \alpha} f(-z)\right)^{\prime}}{\mathcal{N}_{n, m, q}^{\lambda, \alpha} f(z)-\mathcal{N}_{n, m, q}^{\lambda, \alpha} f(-z)}\right)\left(\frac{2 z}{\mathcal{N}_{n, m, q}^{\lambda, \alpha} f(z)-\mathcal{N}_{n, m, q}^{\lambda, \alpha} f(-z)}\right)^{\mu}-\rho\right]\right\} \\
\geq \operatorname{Re} h(z)\left[1-\frac{2|\gamma| m r^{m}}{\mu\left(1-r^{2 m}\right)}\right],|z|=r<1,
\end{gathered}
$$

and the right-hand side of Equation (31) is positive provided that $r<R$, where $R$ is given by Equation (29).

Theorem 7. Let $f \in \mathcal{M}_{n, m, q}^{\lambda, \alpha}(\gamma, \mu, A, B)$, let $\gamma \in \mathbb{C}^{*}$ with $\operatorname{Re} \gamma \geq 0$, and $-1 \leq B<A \leq 1$.

1. Then,

$$
\begin{gathered}
\frac{\mu}{\gamma m} \int_{0}^{1} \frac{1-A u}{1-B u} u^{\frac{\mu}{\gamma m}}-1 d u<\operatorname{Re}\left(\frac{2 z}{\mathcal{N}_{n, m, q}^{\lambda, \alpha} f(z)-\mathcal{N}_{n, m, q}^{\lambda, \alpha} f(-z)}\right)^{\mu} \\
<\frac{\mu}{\gamma m} \int_{0}^{1} \frac{1+A u}{1+B u} u^{\frac{\mu}{\gamma m}-1} d u, z \in \mathbb{U} .
\end{gathered}
$$

2. For $|z|=r<1$, we have

$$
\begin{gathered}
2 r\left(\frac{\mu}{\gamma m} \int_{0}^{1} \frac{1+A u r}{1+B u r} u^{\frac{\mu}{\gamma m}-1} d u\right)^{-\frac{1}{\mu}}<\left|\mathcal{N}_{n, m, q}^{\lambda, \alpha} f(z)-\mathcal{N}_{n, m, q}^{\lambda, \alpha} f(-z)\right| \\
<2 r\left(\frac{\mu}{\gamma m} \int_{0}^{1} \frac{1-A u r}{1-B u r} u^{\frac{\mu}{\gamma m}-1} d u\right)^{-\frac{1}{\mu}} .
\end{gathered}
$$

All these inequalities are the best possible.

Proof. From the assumptions, using Theorem 1, we obtain that

$$
\left(\frac{2 z}{\mathcal{N}_{n, m, q}^{\lambda, \alpha} f(z)-\mathcal{N}_{n, m, q}^{\lambda, \alpha} f(-z)}\right)^{\mu} \prec \Psi(z):=\frac{\mu}{\gamma m} \int_{0}^{1} \frac{1+A z u}{1+B z u} u^{\frac{\mu}{\gamma m}-1} d u,
$$

and the convex function $\Psi \in \mathcal{H}[1, m]$ is the best dominant. Therefore,

$$
\begin{aligned}
& \operatorname{Re}\left(\frac{2 z}{\mathcal{N}_{n, m, q}^{\lambda, \alpha} f(z)-\mathcal{N}_{n, m, q}^{\lambda, \alpha} f(-z)}\right)^{\mu}<\sup _{z \in \mathbb{U}} \operatorname{Re}\left(\frac{\mu}{\gamma m} \int_{0}^{1} \frac{1+A z u}{1+B z u} u^{\frac{\mu}{\gamma m}-1} d u\right) \\
& =\frac{\mu}{\gamma m} \int_{0}^{1} \sup _{z \in \mathbb{U}} \operatorname{Re}\left(\frac{1+A z u}{1+B z u}\right) u^{\frac{\mu}{\gamma m}-1} d u=\frac{\mu}{\gamma m} \int_{0}^{1} \frac{1+A u}{1+B u} u^{\frac{\mu}{\gamma m}-1} d u, z \in \mathbb{U},
\end{aligned}
$$


and

$$
\begin{aligned}
& \operatorname{Re}\left(\frac{2 z}{\mathcal{N}_{n, m, q}^{\lambda, \alpha} f(z)-\mathcal{N}_{n, m, q}^{\lambda, \alpha} f(-z)}\right)^{\mu}>\inf _{z \in \mathbb{U}} \operatorname{Re}\left(\frac{\mu}{\gamma m} \int_{0}^{1} \frac{1-A z u}{1-B z u} u^{\frac{\mu}{\gamma m}-1} d u\right) \\
& =\frac{\mu}{\gamma m} \int_{0}^{1} \inf _{z \in \mathbb{U}} \operatorname{Re}\left(\frac{1-A z u}{1-B z u}\right) u^{\frac{\mu}{\gamma m}-1} d u=\frac{\mu}{\gamma m} \int_{0}^{1} \frac{1-A u}{1-B u} u^{\frac{\mu}{\gamma m}-1} d u, z \in \mathbb{U} .
\end{aligned}
$$

In addition, since

$$
\begin{aligned}
& \left|\frac{2 z}{\mathcal{N}_{n, m, q}^{\lambda, \alpha} f(z)-\mathcal{N}_{n, m, q}^{\lambda, \alpha} f(-z)}\right|^{\mu}<\sup _{z \in \mathbb{U}}\left|\frac{\mu}{\gamma m} \int_{0}^{1} \frac{1+A z u}{1+B z u} u^{\frac{\mu}{\gamma m}-1} d u\right| \\
= & \frac{\mu}{\gamma m} \int_{0}^{1} \sup _{z \in \mathbb{U}}\left|\frac{1+A z u}{1+B z u}\right| u^{\frac{\mu}{\gamma m}-1} d u=\frac{\mu}{\gamma m} \int_{0}^{1} \frac{1+A u r}{1+B u r} u^{\frac{\mu}{\gamma m}-1} d u,|z|=r<1,
\end{aligned}
$$

we get

$$
\left|\mathcal{N}_{n, m, q}^{\lambda, \alpha} f(z)-\mathcal{N}_{n, m, q}^{\lambda, \alpha} f(-z)\right|>2 r\left(\frac{\mu}{\gamma m} \int_{0}^{1} \frac{1+A u r}{1+B u r} u^{\frac{\mu}{\gamma m}-1} d u\right)^{-\frac{1}{\mu}}
$$

while

$$
\begin{gathered}
\left|\frac{2 z}{\mathcal{N}_{n, m, q}^{\lambda, \alpha} f(z)-\mathcal{N}_{n, m, q}^{\lambda, \alpha} f(-z)}\right|^{\mu}>\inf _{z \in \mathbb{U}}\left|\frac{\mu}{\gamma m} \int_{0}^{1} \frac{1-A z u}{1-B z u} u^{\frac{\mu}{\gamma m}-1} d u\right| \\
=\frac{\mu}{\gamma m} \int_{0}^{1} \inf _{z \in \mathbb{U}}\left|\frac{1-A z u}{1-B z u}\right| u^{\frac{\mu}{\gamma m}-1} d u=\frac{\mu}{\gamma m} \int_{0}^{1} \frac{1-A u r}{1-B u r} u^{\frac{\mu}{\gamma m}-1} d u,|z|=r<1,
\end{gathered}
$$

implies

$$
\left|\mathcal{N}_{n, m, q}^{\lambda, \alpha} f(z)-\mathcal{N}_{n, m, q}^{\lambda, \alpha} f(-z)\right|<2 r\left(\frac{\mu}{\gamma m} \int_{0}^{1} \frac{1-A u r}{1-B u r} u^{\frac{\mu}{\gamma m}-1} d u\right)^{-\frac{1}{\mu}} .
$$

The inequalities of Equations (32) and (33) are the best possible because the subordination in Equation (34) is sharp.

Taking $q \rightarrow 1^{-}$in Theorem 7 , we obtain the following corollary:

Corollary 8. Let $f \in \mathcal{W}_{n, m}^{\lambda, \alpha}(\gamma, \mu, A, B)$, let $\gamma \in \mathbb{C}^{*}$ with $\operatorname{Re} \gamma \geq 0$, and $-1 \leq B<A \leq 1$.

1. Then,

$$
\begin{gathered}
\frac{\mu}{\gamma m} \int_{0}^{1} \frac{1-A u}{1-B u} u^{\frac{\mu}{\gamma m}-1} d u<\operatorname{Re}\left(\frac{2 z}{\mathcal{I}_{n, m}^{\lambda, \alpha} f(z)-\mathcal{I}_{n, m}^{\lambda, \alpha} f(-z)}\right)^{\mu} \\
<\frac{\mu}{\gamma m} \int_{0}^{1} \frac{1+A u}{1+B u} u^{\frac{\mu}{\gamma m}-1} d u, z \in \mathbb{U} .
\end{gathered}
$$


2. For $|z|=r<1$, we have

$$
\begin{gathered}
2 r\left(\frac{\mu}{\gamma m} \int_{0}^{1} \frac{1+A u r}{1+B u r} u^{\frac{\mu}{\gamma m}-1} d u\right)^{-\frac{1}{\mu}}<\left|\mathcal{I}_{n, m}^{\lambda, \alpha} f(z)-\mathcal{I}_{n, m}^{\lambda, \alpha} f(-z)\right| \\
<2 r\left(\frac{\mu}{\gamma m} \int_{0}^{1} \frac{1-A u r}{1-B u r} u^{\frac{\mu}{\gamma m}-1} d u\right)^{-\frac{1}{\mu}} .
\end{gathered}
$$

All these inequalities are the best possible.

Taking $q \rightarrow 1^{-}, \alpha=0$ and $\lambda=1$ in Theorem 7 , we obtain the following corollary:

Corollary 9. Let $f \in \mathcal{N}^{\gamma, \mu}(m, A, B)$, let $\gamma \in \mathbb{C}^{*}$ with $\operatorname{Re} \gamma \geq 0$, and $-1 \leq B<A \leq 1$.

1. Then,

$$
\frac{\mu}{\gamma m} \int_{0}^{1} \frac{1-A u}{1-B u} u^{\frac{\mu}{\gamma m}-1} d u<\operatorname{Re}\left(\frac{2 z}{f(z)-f(-z)}\right)^{\mu}<\frac{\mu}{\gamma m} \int_{0}^{1} \frac{1+A u}{1+B u} u^{\frac{\mu}{\gamma m}-1} d u, z \in \mathbb{U} .
$$

2. For $|z|=r<1$, we have

$$
\begin{gathered}
2 r\left(\frac{\mu}{\gamma m} \int_{0}^{1} \frac{1+A u r}{1+B u r} u^{\frac{\mu}{\gamma m}-1} d u\right)^{-\frac{1}{\mu}}<|f(z)-f(-z)| \\
<2 r\left(\frac{\mu}{\gamma m} \int_{0}^{1} \frac{1-A u r}{1-B u r} u^{\frac{\mu}{\gamma m}-1} d u\right)^{-\frac{1}{\mu}} .
\end{gathered}
$$

All these inequalities are the best possible.

Example 4. Putting $\mu=\gamma=m=1, A=1-2 \beta(0 \leq \beta<1)$, and $B=-1$ in Corollary 9, we get the next special case.

If $f \in \mathcal{N}^{1,1}(1,1-2 \beta,-1)$ with $0 \leq \beta<1$, then:

1. The next inequality holds:

$$
\operatorname{Re} \frac{2 z}{f(z)-f(-z)}>2 \beta-1+2(1-\beta) \ln 2, z \in \mathbb{U} .
$$

2. For $|z|=r:=0.9$, we have

$$
\frac{1.8}{1+3.116855762 \beta}<|f(z)-f(-z)|<\frac{1.8}{1-0.5736580307 \beta} .
$$

Remark 2. Part (ii) of Corollary 9 corrects the Corollary (3.10) studied by Muhammad and Marwan [16].

Concluding, all the above results give us information about subordination and superordination properties, inclusion results, radius problem, and sharp estimations for the classes $\mathcal{M}_{n, m, q}^{\lambda, \alpha}(\gamma, \mu, A, B)$, together general sharp subordination and superordination for the operator $\mathcal{N}_{n, m, q}^{\lambda, \alpha}$. For special choices of the parameters $\gamma \in \mathbb{C}, 0<\mu<1,-1 \leq B<A \leq 1, m \in \mathbb{N}, \alpha>0, n \geq 0,0<q<1$, and $\lambda>-1$, we may obtain several simple applications connected with the above-mentioned classes and operator.

Author Contributions: The authors contributed equally to this work. 
Funding: This research received no external funding.

Acknowledgments: The authors are grateful to the reviewers of this article, who gave valuable remarks, comments, and advice, in order to revise and improve the results of the paper.

Conflicts of Interest: The authors declare no conflict of interest.

\section{References}

1. Jackson, F.H. On $q$-functions and a certain difference operator. Trans. R. Soc. Edinb. 1909, 46, $253-281$. [CrossRef]

2. Jackson, F.H. On q-definite integrals. Quart. J. Pure Appl. Math. 1910, 41, 193-203.

3. Abu-Risha, M.H.; Annaby, M.H.; Ismail, M.E.; Mansour, Z.S. Linear q-difference equations. Z. Anal. Anwend. 2007, 26, 481-494. [CrossRef]

4. Bulboacă, T. Differential Subordinations and Superordinations. Recent Results; House of Scientific Book Publ.: Cluj-Napoca, Romania, 2005.

5. Miller, S.S.; Mocanu, P.T. Differential Subordinations. Theory and Applications; Series on Monographs and Textbooks in Pure and Applied Mathematics; Marcel Dekker Inc.: New York, NY, USA; Basel, Switzerland, 2000; Volume 225.

6. Miller, S.S.; Mocanu, P.T. Subordinants of differential superordinations. Complex Var. 2003, 48, 815-826. [CrossRef]

7. Bulboacă, T. A class of superordination-preserving integral operators. Indag. Math. 2002, 13, 301-311. [CrossRef]

8. Bulboacă, T. Classes of first order differential superordinations. Demonstr. Math. 2002, 35, 287-292. [CrossRef]

9. Aouf, M.K.; Al-Oboudi, F.M.; Haidan, M.M. On some results for $\lambda$-spirallike and $\lambda$-Robertson functions of complex order. Publ. Inst. Math. 2005, 75, 93-98. [CrossRef]

10. Aouf, M.K.; Bulboacă, T. Subordination and superordination properties of multivalent functions defined by certain integral operator. J. Frankl. Inst. 2010, 347, 641-653. [CrossRef]

11. Ali, R.M.; Ravichandran, V.; Hussain Khan, M.; Subramanian, K.G. Differential sandwich theorems for certain analytic functions. Far East J. Math. Sci. 2004, 15, 87-94.

12. Sakaguchi, K. On certain univalent mapping. J. Math. Soc. Jpn. 1959, 11, 72-75. [CrossRef]

13. Muhammad, A. Some differential subordination and superordination properties of symmetric functions. Rend. Semin. Mat. Univ. Politec. Torino 2011, 69, 247-259.

14. Aouf, M.K.; El-Ashwah, R.M.; El-Deeb, S.M. Certain classes of univalent functions with negative coefficients and $n$-starlike with respect to certain points. Mat. Vesn. 2010, 62, 215-226.

15. Sălăgean, G.S. Subclasses of univalent functions. In Lecture Notes in Math; Springer: Berlin/Heidelberg, Germany, 1983; Volume 1013, pp. 362-372.

16. Muhammad, A.; Marwan, M. Some properties of generalized two-fold symmetric non-Bazilević analytic functions. Matematiche 2014, 69, 223-235.

17. Shanmugam, T.N.; Ravichandran, V.; Sivasubramanian, S. Differential sandwich theorems for some subclasses of analytic functions. Aust. J. Math. Anal. Appl. 2006, 3, 1-11.

18. Liu, M.S. On certain subclass of analytic functions. J. South China Norm. Univ. Natur. Sci. Ed. 2002, 4, 15-20.

19. Shah, G.M. On the univalence of some analytic functions. Pac. J. Math. 1972, 43, 239-250. [CrossRef]

20. MacGregor, T.H. The radius of univalence of certain analytic functions. Proc. Am. Math. Soc. 1963, 14, 514-520. [CrossRef]

(C) 2019 by the authors. Licensee MDPI, Basel, Switzerland. This article is an open access article distributed under the terms and conditions of the Creative Commons Attribution (CC BY) license (http://creativecommons.org/licenses/by/4.0/). 